IdeAs

Idées d'Amériques

$3 \mid 2012$

L'alimentation dans les Amériques au prisme des sciences sociales

\title{
La redéfinition des plats et des pratiques alimentaires populaires au Mexique
}

La redefinición de platillos y prácticas populares en México

The Redefinition of Popular Dishes and Dietary Practices in Mexico

Domingo Garcia-Garza

OpenEdition

Journals

Édition électronique

URL : https://journals.openedition.org/ideas/461

DOI : 10.4000/ideas.461

ISSN : 1950-5701

Éditeur

Institut des Amériques

Référence électronique

Domingo Garcia-Garza, "La redéfinition des plats et des pratiques alimentaires populaires au

Mexique », IdeAs [En ligne], 3 | 2012, mis en ligne le 03 janvier 2013, consulté le 18 octobre 2022. URL http://journals.openedition.org/ideas/461 ; DOl : https://doi.org/10.4000/ideas.461

Ce document a été généré automatiquement le 18 octobre 2022.

Creative Commons - Attribution - Pas d'Utilisation Commerciale - Pas de Modification 4.0 International - CC BY-NC-ND 4.0

https://creativecommons.org/licenses/by-nc-nd/4.0/ 


\title{
La redéfinition des plats et des pratiques alimentaires populaires au Mexique
}

\author{
La redefinición de platillos y prácticas populares en México \\ The Redefinition of Popular Dishes and Dietary Practices in Mexico
}

Domingo Garcia-Garza

\section{Introduction}

1 Perçue au départ comme une pratique alimentaire propre aux groupes sociaux

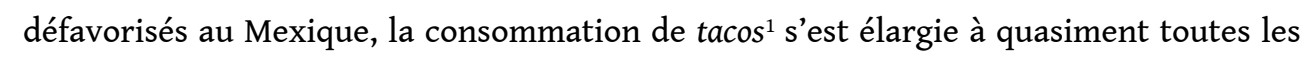
catégories sociales au cours du XX⿳亠े⿵冂丶 ${ }^{2}$ siècle au point de devenir, de la même façon que la samba au Brésil, un «emblème national » (Rivron, 2010). Elle est devenue l'une des références du système alimentaire mexicain qui, à l'instar d'autres expressions culinaires mexicaines (Pilcher, 1998), joue un rôle de vecteur de l'identité nationale (Bruegel \& Laurioux, 2002). Comment se fait-il que cette façon de consommer les aliments se soit généralisée dans tout l'espace social? Comment se construit socialement cette légitimité des pratiques alimentaires d'origine populaire?

2 Cette forme de consommation marchande s'est progressivement propagée dans les différents milieux sociaux grâce à l'influence de deux processus en apparence opposés, mais complémentaires : la patrimonialisation ${ }^{2}$ des pratiques culinaires nationales et l'«internationalisation" de ces mêmes pratiques (Garcia-Garza, 2010). Ces deux influences auraient changé la perception de ce type d'alimentation aux yeux des consommateurs, alors qu'elle symbolisait jusqu'au $\mathrm{XX}^{\text {ème }}$ siècle tous les stigmates de la «classe populaire» (petits plats de pauvres, consommation de rue, absence de couverts, etc.). Tout d'abord, la banalisation dont la consommation de tacos a fait l'objet s'appuie sur un registre de légitimité culturelle valorisant les pratiques issues $\mathrm{du}$ peuple, supposées véhiculer une certaine "authenticité». Or, les tacos ont ensuite bénéficié également d'une notoriété internationale. De la même manière que d'autres 
biens culturels d'origine latino-américaine, la revalorisation des tacos s'est opérée plus facilement par des consommateurs, souvent étrangers, qui ne partagent pas les mêmes catégories de perception que les Mexicains et sont davantage à la recherche d'« exotisme». La représentation qu'ont les Mexicains des tacos a changé de par leur diffusion aux États-Unis (Pilcher, 2008 ; Arellano, 2012) et dans le monde (Pilcher, 2012 : 11).

3 La redéfinition des plats et des pratiques populaires ressemble à celle dont a fait l'objet la pizza (Sanchez, 2007). Ayant acquis un nouveau statut social, les tacos se sont diffusés dans l'ensemble de la société. Cette diffusion dans tout l'espace social constitue un processus historique qui permet de comprendre la manière dont se construit socialement une tradition nationale. Ce processus s'est amorcé grâce à la médiation de différents acteurs sociaux, jeunes consommateurs et marchands de tacos, qui rend possible l'existence d'un tel marché: les premiers en diffusant ce type de consommation alimentaire au-delà du monde de la rue, et les seconds en réduisant les marges de qualité à un seuil socialement acceptable (Garcia-Garza, 2011). On peut dire que les tacos se sont « embourgeoisés » dans la mesure où leur consommation est partie du bas de la pyramide sociale jusqu'à s'étendre vers le haut, couvrant ainsi l'ensemble de la société.

4 Notre hypothèse est la suivante : l'anoblissement des tacos s'est fait au prix d'une montée en gamme par rapport aux contenus et aux lieux où ils sont consommés. Née dans les classes populaires, la consommation des tacos a été adoptée, mais surtout adaptée par d'autres groupes sociaux à modes de consommation plus modernes. La grande majorité des chercheurs travaillant sur la question s'accordent à dire que les tacos, tels qu'on les connaît aujourd'hui, et contrairement à ce que l'on peut penser, correspondent à une « invention de la tradition» (Hobsbawm \& Ranger, 2006). Nous défendons la thèse que cette manière de consommer les aliments est une invention de la modernité (Pilcher, 2006), voire même une tradition inventée par la modernité, qui se serait constituée de façon informelle et non concertée dans les différentes régions. À travers le cas de la ville de Monterrey (nord-est du Mexique), nous essayerons de montrer que cette tradition a été ajustée, grâce aux garnitures (viandes, légumes, insectes, etc.), aux différents milieux sociaux et à leurs cadres respectifs de consommation.

5 Nous esquisserons ainsi une première typologie des tacos à la lumière de pratiques sociales traditionnelles ( $1^{\mathrm{e}}$ partie) et une seconde résultant des mutations ultérieures entrainées par la modernité ( $2^{\mathrm{e}}$ partie). Ensuite nous saisirons l'évolution qualitative des garnitures ( $3^{\mathrm{e}}$ partie) et les nouvelles scènes sociales ou lieux de consommation dans lesquelles elle va naître ( $4^{\mathrm{e}}$ partie). Cela nous permettra de mettre en lumière la façon dont la légitimité des pratiques culturelles, processus dans lequel l'alimentation se retrouve redéfinie, et particulièrement celle des tacos qui constitue un cas exemplaire.

\section{Méthodologie et terrain}

Cet article est le prolongement d'une recherche ethnographique qui a donné lieu à une thèse de sociologie soutenue à l'EHESS en 2009. La méthode d'enquête choisie est l'ethnographie combinant l'observation, l'entretien et l'analyse de cas. Cette étude est le résultat d'une série d'enquêtes de terrain, étalée sur une période de cinq ans et menée à Monterrey au Mexique. Dans un premier temps, j'ai mené des 
entretiens semi-directifs auprès de vendeurs de tacos et de consommateurs directement sur les stands de rue et dans les restaurants. Dans un deuxième temps, j'ai effectué des observations ethnographiques auprès d'une cinquantaine de marchands ambulants et d'une quinzaine de restaurants. J'ai réalisé ces entretiens et ces observations entre 2006 à 2008, au cours de trois séjours de terrain de quatre mois chacun. Les dernières données ont été collectées dans un troisième temps lors de deux courtes visites en décembre-janvier 2009 et en octobre 2012. L'étalement de l'enquête sur une durée de 5 ans a ainsi permis de rendre compte de l'évolution qualitative qu'a connue le marché des tacos dans le temps.

\section{La typologie traditionnelle des tacos selon les positions sociales}

6 Les tortillas agrémentées d'une garniture, même la plus simple, prennent le nom de «taco». Les tacos bénéficient du statut de produit "générique» d'une grande versatilité. Ils peuvent être préparés un peu partout et avec différentes garnitures. Cette plasticité permet à toutes les singularités régionales d'imprimer leur sensibilité propre en même temps qu'elles participent à définir l'identité culinaire nationale. Le support de maiis, au départ un complément de la diète quotidienne, est devenu un élément fondamental de la cuisine dite " populaire » (les tortillas et les sauces à base de tomate et piment sont toujours à table au Mexique). Les tacos même les plus économiques constituent également un apport de calories important, par exemple ceux garnis aux haricots, qui, combinés avec le maïs, sont très riches en protéines. Le faible coût des garnitures généralement utilisées et leur teneur protéinique ont permis à beaucoup de ménages de subvenir à leurs besoins, que ce soit comme source de revenus en les vendant, ou soit comme remplacement alimentaire en les achetant. Pour ces raisons, les tacos souffrent de divers stigmates et sont associés depuis longtemps aux groupes sociaux défavorisés. Les stigmates les plus courants sont ceux rattachés à la consommation de maïs (historiquement associée aux indigènes), aux bas prix (qui renvoient aux groupes sociaux défavorisés ou plus largement à la pauvreté) et à la saleté (liée à la consommation dans la rue). L'aversion pour la mixité sociale (par la présence de consommateurs de classes sociales inférieures) des lieux de consommation contribue également à ternir l'image des tacos.

7 Or, les tacos ont connu un processus d'enrichissement de garnitures et de diversification d'ingrédients très important depuis une dizaine d'années. Ce processus n'est pas très récent, mais il s'est particulièrement accentué au cours du XX ${ }^{\text {ème }}$ siècle. Cependant, l'évolution des plats n'a pas entraîné une modification immédiate des pratiques alimentaires. Le syncrétisme culinaire a été un processus culturel relativement lent. Il s'est traduit d'abord par un changement de plats qui a ensuite induit une modification des pratiques alimentaires et des formes d'alimentation. Le décalage entre la préparation de la nourriture et sa consommation s'explique essentiellement par des facteurs sociaux (baisse du pouvoir d'achat, modification d'habitudes alimentaires, quête de différenciation sociale, identité nationale, etc.). À l'origine, l'usage des garnitures «bas de gamme » a sans doute été le premier obstacle à l'adoption des tacos par la « bonne société ». L'entrée relativement récente des tacos au restaurant et l'apparition de nouvelles modalités de vente de tacos, plus conformes aux normes de restauration traditionnelle, ont favorisé leur consommation par les groupes sociaux aisés. 
On peut établir un premier classement en fonction de la garniture que l'on ajoute aux tacos (insectes, féculents, abats, viandes, poissons, etc.), étroitement liée aux ressources économiques et le système de préférence de chaque groupe social. En bas de la pyramide sociale, on trouve le plus souvent des tacos garnis d'abats, d'insectes, de légumes (ou de féculents) tandis que les groupes plus favorisés privilégient la viande et le poisson. Néanmoins, les transformations récentes du marché alimentaire, le relâchement des comportements de consommation et l'apparition des nouveaux styles de vie ont rendu cette classification plus flottante. Les nouveaux types de tacos correspondent aux transformations des styles de vie, et bien évidemment, à l'évolution $\mathrm{du}$ pouvoir d'achat et des pratiques alimentaires qui lui sont associées. "Manger dehors » n'est plus stigmatisé comme dans le passé. Aujourd'hui, c'est une source de plaisir, de différenciation et de gratification (Warde \& Martens, 2000), voire de distinction. Il en résulte que les pratiques de consommation dépendent aussi bien de la manière de consommer de chaque groupe d'appartenance que de la composition des tortillas et de la scène sociale (moments et lieux) dans laquelle la prise alimentaire intervient, ainsi que d'un rapport distancié aux pratiques culturelles. Car si leur consommation s'est propagée à tous les groupes sociaux, les écarts qui les différencient se maintiennent, ce qui renvoie aux analyses sur la consommation culturelle qui établissent que la manière de consommer les produits est au moins aussi différenciée que les biens consommés (Bourdieu, 1979).

9 Adaptés aux goûts de différents groupes sociaux, les tacos se consomment de manière ritualisée sans ustensiles de table particuliers, directement avec les mains. De ce fait, les tacos sont considérés comme un plat populaire, circonscrit à l'espace domestique ou réservé au monde de la rue (travailleurs, ouvriers, livreurs, chauffeurs de taxi, etc.). Les taquerias sont des petits " commerces de rue ", qui prennent le plus souvent la forme de stands « informels ». Le commerce ambulant dont font partie les vendeurs de tacos est une activité économique produite par la mobilité spatiale entrainée elle-même par le phénomène de la «métropolisation » (Monnet, 2006b). Il est indispensable de souligner le rôle joué par le processus d'urbanisation accélérée qui a caractérisé les grandes villes mexicaines à partir des années 1940. C'est grâce à ce processus que sont nées l'externalisation et la marchandisation de certaines pratiques de la vie quotidienne telles que l'alimentation. Les marchands de tacos vont combler les "trous » créés par l'urbanisation dans la chaîne de distribution. C'est grâce aux nouvelles dynamiques métropolitaines induites par l'intensification du flux de personnes et de biens que le marché des tacos a vu le jour. Les marchands « ambulants » (Monnet, 2006a) sont ainsi les premiers à avoir commercialisé les tacos. Leur commerce constitue une réponse aux nouveaux besoins urbains et alimentaires des usagers de l'espace public. Dans sa version marchande, ce mode d'alimentation est resté un repas «hors domicile» : la manière de consommer les tacos est passée du repas pris «sur le pouce » à la taqueria ${ }^{3}$ établie dans un local commercial, pour arriver enfin dans les années 1990 au restaurant (où ils sont servis généralement dans une assiette avec la tortilla déposée à plat sous la garniture). L'entrée de ce met et de ces pratiques alimentaires au « restaurant » a agi comme une « instance de légitimation » qui a changé la perception sociale des tacos en redéfinissant leur statut, et par là la hiérarchie alimentaire.

Entre-temps, des taquerias pour les « jeunes», sont apparues. Celles-ci sont une version à mi-chemin entre la taqueria populaire et le restaurant de tacos. Cette étape intermédiaire de pérennisation des lieux et la formalisation des plats et des pratiques 
sont nées de la dynamique festive qui s'est déclenchée dans le vieux quartier du centreville à Monterrey appelé barrio antiguo (aujourd'hui disparu à cause du narcotrafic). Les consommations nocturnes de tacos se sont diffusées à partir des années 1990 et accompagnent, sur le plan alimentaire, les « sorties » des jeunes. Les taquerias ciblant ce groupe d'âge sont apparues parallèlement aux nouveaux espaces de socialisation des jeunes appelés antros (l'équivalent au Mexique de boîtes de nuit). Le relâchement des mœurs et des habitudes alimentaires s'est renforcé par la présence d'étudiants venus d'autres régions du Mexique à Monterrey, qui est un centre universitaire important du pays. Plus économiques, moins formelles et plus décontractées que les restaurants, les taquerias s'adaptent parfaitement aux nouveaux styles de vie adoptés par les jeunes. Les étudiants, les jeunes « fêtards » et autres noctambules de moins de 40 ans se montrent plus enclins à s'affranchir des conventions sociales qui régissent la consommation, d'autant plus qu'ils sont éloignés du contrôle parental. De ce fait, ils contribuent à diffuser les pratiques d'alimentation populaires et locales au-delà de leur milieu d'origine. Les points de vente d'aliments préparés, dont les tacos sont l'expression la plus emblématique au Mexique, se sont d'abord développés à proximité de lieux de concentration d'activités (économiques, professionnelles, festives), et non pas de façon dispersée.

11 En plus de modifier les pratiques de consommation sur le plan national, les règles de bienséance et les bonnes manières, les tacos ont également pour propriété de ne pas se consommer nécessairement à table. Ils se consomment selon la tradition debout dans la rue ou sur le trottoir (d'où le nom tacos parados), ou même sur le lieu de travail (le champ, le chantier, l'atelier). Mais contrairement aux sandwichs, ils ne se consomment pas en marchant dans la rue. Les tortillas ne sont pas un plat en soi, mais un support d'aliments. En effet, les tacos sont une façon de transporter et de conserver la nourriture. Suivant le même principe que le shawarma (de Suremain, 2008) ou la pizza (Sanchez, 2007), les tacos sont devenus l'un des supports les plus pratiques pour consommer des aliments. Cette transformation ne se diffusera au Mexique qu'après la Révolution Mexicaine (1910-1920). En effet, consommer la nourriture sous forme de tacos ne s'est popularisé sur le territoire mexicain qu'à partir des années 1920, notamment grâce à la diffusion des chemins de fer (Pilcher, 2006). La taqueria révolutionnaire et le restaurant à tacos peuvent être considérés comme l'expression d'un relâchement des comportements et la modification d'habitudes alimentaires qui donnent naissance à de nouveaux types de consommation et de nouveaux styles de vie (Garcia-Garza, 2010).

\section{Nouvelle typologie des tacos selon les pratiques sociales}

Les tacos ne sont devenus des produits commercialisés à grande échelle que tardivement. Leur consommation s'est généralisée au Mexique à partir de la deuxième moitié du XX ${ }^{\text {ème }}$ siècle grâce à l'industrialisation tardive des tortillas (Pilcher, 1998, 2006, 2012; Bauer, 1990, 2001; Novelo \& Garcia, 1987; Aboites, 1989). Celles-ci, par une division sexuée du travail, étaient faites à la main par des femmes grâce à l'ancienne technique (nixtamalización), ce qui par ailleurs représentait pour elles une lourde corvée. Cependant, aujourd'hui certains restaurants de tacos proposent des tortillas industrielles. Les galettes à l'ancienne sont ainsi devenues plus rares et un produit de luxe, car elles demandent une main d'œuvre trop coûteuse et prennent plus de temps à réaliser. Dans tous les cas, la production de tortillas à l'échelle industrielle donne une 
forte impulsion au marché des tacos. Ce type de consommation s'est donc popularisé grâce aux innovations modernes. Cependant, la consommation des tacos n'est pas identique dans tous les milieux. Celle-ci varie selon la position sociale des consommateurs, l'endroit où elle s'effectue et la signification donnée à chaque prise. Situés au carrefour social, les groupes intermédiaires sont ceux qui ont accéléré la banalisation de ces pratiques par l'adoption de nouveaux styles de vie plus décontractés.

De manière analogue à l'évolution que les tacos ont suivie dans la ville de Mexico (Pilcher, 2006), les taquerias sont devenues de nouveaux espaces de socialisation pour les citadins, anciens et nouveaux, de la ville de Monterrey. Le dynamisme économique de la ville a attiré un flux important de paysans du nord-est accompagnés de leurs traditions culinaires régionales; notamment les tacos de barbacoa et de pomme de terre ou dorados. Ces transferts culturels ont contribué à forger une nouvelle cuisine locale. Mais ils induisent aussi des bouleversements sociaux importants, notamment l'assouplissement progressif des habitudes alimentaires. En effet, l'industrialisation de la ville a contribué au relâchement des contrôles sociaux et, s'agissant des pratiques alimentaires, à les sortir de la sphère privée vers la sphère publique. L'essor de petits points de distribution d'aliments s'explique en partie par les difficultés d'approvisionnement en matières premières et par l'insuffisance d'infrastructures de restauration (locaux, services, tables, chaises); sans oublier les coûts élevés de la restauration classique (onéreux pour les revenus les plus faibles). C'est à partir des années 1950-60 que les petites échoppes de tacos dans la rue se multiplient à Monterrey. La population a désormais accès à des produits bon marché pour se nourrir. Un public hétérogène s'approprie l'espace urbain, favorisant une mixité sociale jusquelà inconnue. La singularité de ces nouveaux espaces sociaux réside dans le fait qu'ils sont fréquentés simultanément par des personnes appartenant à différentes classes sociales qui adoptent de nouvelles manières de manger. Aux anciens clients d'origine populaire s'ajoutent désormais les bourgeois, les bourgeois-bohèmes et d'autres catégories sociales qui jusque là ne consommaient pas de tacos dans les taquerias.

Le fait que les premiers à préparer et à consommer les tacos soient essentiellement des personnes issues des communautés de migrants internes ne doit rien au hasard. Parachutés dans les grandes villes, les nouveaux citadins venus des campagnes voisines de Monterrey n'ont pas les compétences professionnelles exigées par le monde urbain. En décalage avec les exigences professionnelles du monde du travail contemporain, beaucoup de migrants ont trouvé dans la vente de tacos une activité économique rentable. La vente d'aliments se révèle à la fois être un métier dont ils maitrisent le savoir-faire, et une activité économique prête à absorber les excédents de maind'œuvre peu qualifiée et peu scolarisée. Dans tous les cas, il s'agit pour la plupart d'une activité dont le coût d'opportunité est plus élevé que celui des activités les plus dévalorisées et les plus précaires de l'économie formelle auxquelles cette population avait généralement accès. Par conséquent, c'est dans l'économie informelle que sont nées les premières taquerias. C'est pourquoi les premiers vendeurs de tacos peuvent être associés à ce que l'on appelle aujourd'hui les travailleurs « informels».

15 À l'origine existait une proximité sociale entre acheteurs et vendeurs de tacos. Appartenant au même monde, les marchands offraient des produits alimentaires qui correspondaient aux habitudes alimentaires de ceux qui venaient comme eux du même terroir. Ils partageaient le même système de préférences culinaires et le même seuil de 
qualité. Les principales garnitures des tacos les plus élémentaires sont pratiquement les mêmes partout au Mexique : des œufs, des pommes de terre, des haricots, des herbes sauvages (quelites), des abats (tripes), de la viande de porc frite (rillons, couenne, carnitas), etc. Ce type de tacos est le moins coûteux, et le plus riche en protéines, c'est pourquoi il est consommé notamment par les groupes à faibles revenus. Cela ne veut pas dire pour autant que ces garnitures à bas prix, longtemps considérées comme "populaires ", n'aient pas de goût, ou que le goût soit forcément "de nécessité ». L'usage de ces garnitures renvoie plutôt à un attachement pour les produits « de la terre » ou de la campagne. Le goût recherché est justement celui du terroir ou de la cuisine "faite maison». L'effet de distinction qui émane de ce type de consommation dépend alors du rapport des groupes sociaux favorisés aux plats et aux pratiques gastronomiques populaires (campagnardes ou domestiques) et particulièrement à leur distance à la nécessité (Bourdieu, 1979: 198). Un effet de distinction supplémentaire découle $d u$ fait que la consommation des plats, considérés comme authentiques, renvoie au nationalisme revendiqué historiquement par les élites mexicaines (Pilcher, $2012: 81)$.

\section{Les nouvelles garnitures des tacos}

La revalorisation de cette pratique domestique et populaire s'est faite parallèlement à des transformations plus larges. L'engouement contemporain pour les tacos provient notamment des nouvelles variétés de garnitures proposées. Celles-ci sont passées des assaisonnements les plus simples (œufs, patates, haricots) aux mets de style "gourmet». Pour ne citer que quelques exemples, parmi les nouvelles garnitures figurent les insectes comme les chenilles (chinicuiles), des larves de fourmis (escamoles), et sauterelles (chapulines); divers légumes extravagants comme le cuitlacoche ${ }^{4}$, des poissons et fruits de mer (merlin, tilapia, langoustes, crevettes) et des morceaux de choix pour les viandes (dont nous présenterons la hiérarchie plus loin). L'usage de ces nouvelles garnitures représente une modification substantielle et inédite du contenu. Elles représentent surtout un saut qualitatif. Ce saut est d'autant plus significatif que les tacos sont habituellement préparés avec des produits «bas de gamme ». En ce sens, les nouveaux tacos n'ont rien, ou peu à voir, avec les versions dites «traditionnelles » des tacos.

Les tacos s'inscrivent dans un processus de différenciation sociale plus large. La panoplie des garnitures s'est élargie progressivement à des mets qui normalement n'étaient pas destinés à la mise en galette. On voit ainsi apparaitre à l'aube du XX ${ }^{\text {ème }}$ siècle sur le marché des tacos des garnitures d'origine paysanne. Il s'agit essentiellement de ragoûts plus sophistiqués que ceux des versions d'origine. Il faut y voir une manière d'échapper à la concurrence en diversifiant l'offre, mais aussi une stratégie qui vise à élargir la réception de ces biens. Le marché des tacos ressemble en ce sens à celui du café ou à celui du vin dans la mesure où les marchands, pour s'étendre sur un marché saturé, jouent la carte de la différenciation des produits (montée en gamme, déclinaisons, sophistication) et visent la conquête de nouveaux marchés (niches, quartiers, villes). La diversification de l'offre a redéfini la hiérarchie alimentaire quant à ce produit et a ainsi permis de déclencher sur une période très courte une nouvelle demande. 
18 Au cours des dix dernières années en effet, on a ainsi vu apparaître sur le marché local un nombre grandissant d'établissements de tacos. Ceci est une réponse au ralentissement du marché alimentaire traditionnel tout en intégrant les logiques du marché des fast foods en pleine expansion depuis les années 2000. La tendance de ces nouveaux marchands est de proposer des tacos haut de gamme. À l'instar du marché de rue, l'enjeu pour les restaurants de tacos porte moins sur les prix que sur la qualité organoleptique des produits proposés (Garcia-Garza, 2009). La différence est que les restaurants proposent une variété de garnitures peu fréquentes sur le marché. Ces garnitures sont soit transposées de la cuisine gastronomique étrangère, soit inspirées de la cuisine domestique, cette dernière s'inspirant à son tour du terroir. Les tacos n'échappent donc pas à la règle selon laquelle la cuisine contemporaine résulte d'une part, d'une nouvelle interprétation des traditions culinaires issues du terroir, et d'autre part, de l'exotisme venu de l'étranger.

19 On voit ainsi apparaître à Monterrey de nouveaux restaurants de tacos. L'une des stratégies la plus couramment utilisée consiste à introduire des garnitures à base de ragoûts traditionnels. Dans ce premier groupe, on distingue deux groupes de marchands : les étals de rue et les restaurants. On trouve dans la catégorie des «étals » à Monterrey par exemple: Tacos Chava, Tacos Lacho, Tacos El Fer. Et, dans la catégorie des «restaurants»: Tacos Mode et Tacos Leal. Les deux types d'établissements proposent une offre légèrement élargie de tacos à base de ragoûts inspirés de la cuisine populaire mexicaine. Cependant, cette offre est aussi standardisée et se limite aux garnitures suivantes : chicharrón (rillons), desherbrada (viande déchiquetée) et picadillo (ragoût à base de viande hachée bas de gamme). D'autres marchands, ciblant une clientèle plus exclusive, proposent les mêmes garnitures, mais dans une version plus élaborée. Le stand de Tacos $\mathrm{El} \mathrm{Fer} \mathrm{propose} \mathrm{par} \mathrm{exemple} \mathrm{des} \mathrm{tacos} \mathrm{de} \mathrm{:} \mathrm{barbacoa} \mathrm{(viande}$ de bœuf cuite à la vapeur), steak au chipotle (une variété de piment), chicharrón (rillons) nature ou à la sauce rouge et/ou verte, viande de porc au piment morita, rajas con queso (piment au fromage), arrachera (bavette) grillée et piment farci (une déclinaison des fameux chiles rellenos traditionnels appartenant à la cuisine gastronomique).

Un deuxième groupe d'échoppes de tacos décline une offre à base de morceaux de viande grillée. Dans la rue, on trouve de nombreux exemples comme "Tacos Lucas", "Tacos Madero», etc. Et parmi les restaurants, les exemples sont multiples: «El Buey », «El Papalote Taco \& Grill », «Papa Bill's Saloon », « Tacos \& Salsa Uuf !!! ». La différence entre les deux, au-delà des installations, est la qualité de la viande. Dans la rue, on trouve généralement des garnitures à base de viande grillée bas de gamme, par exemple le bifteck du numéro zéro dit clôt; et les restaurants établis offrent plutôt des morceaux de choix plus coûteux parmi lesquels figurent l'arrachera (bavette), le sirloin (faux-filet), le tenderloin (filet-mignon) et le rib-eye (entrecôte). Les stands de rue sont installés en général en centre-ville et sont fréquentés par une population essentiellement masculine. Les restaurants à tacos se trouvent plutôt dans les quartiers résidentiels de la périphérie et sont fréquentés par des jeunes couples et des familles de milieux plus aisés. On s'aperçoit que le marché de tacos se dessine selon la géographie sociale de la ville.

21 Le troisième groupe constitue une grande nouveauté pour le marché local situé dans une zone semi-désertique: les tacos au poisson. La stratégie de ces établissements consiste à préparer des tacos dont la garniture est à base de poisson (bon marché) par exemple le merlin ou la tilapia, ou plutôt des beignets de poisson et/ou de fruits de mer 
(crevettes, poulpe, calamar). Cette dernière stratégie est, semble-t-il, la plus porteuse. Elle consiste à préparer à la façon des tacos la cuisine « de mer ", notamment celle de la côte ouest (en générale de l'État de Sinaloa). Récemment, le nombre de ces restaurants a augmenté de manière significative. Les premières échoppes de ce type sont les Tacos Meiji-en qui apparaissent en 2005, suivie par « El Torito Sinaloense " peu de temps après (2007). Les établissements pionniers de tacos de poisson correspondent plus à une offre informelle assimilée au stand de rue ou au bar de plage. Ces boutiques ont connu un succès rapide et ont initié la tendance de ce type de tacos. Les consommateurs sont en général d'origine sociale aisée. Par contre, El Muelle (2007), Cabo Grill (2008) et La Proa (2010) sont plutôt de véritables restaurants qui proposent une déclinaison de la cuisine de mer sous forme de tacos. La Barquera (2010) correspond à la version fast food des tacos. Les établissements précédents sont apparus récemment et les consommateurs sont là aussi d'origine sociale plus aisée. Leur clientèle demeure cependant hétérogène. Enfin, le quatrième et dernier type de tacos est plus sophistiqué : les tacos d'insectes. Les tacos d'insectes se veulent une revalorisation de la cuisine précolombienne. Ils étaient réservés jusqu'à très récemment aux groupes ethniques minoritaires et socialement défavorisés du centre et du sud du pays. Dans cette catégorie l'offre est limitée aux restaurants comme La Catarina et La Félix. Tous deux appartiennent à la même entreprise (Grupo Pangea) spécialisée dans la cuisine "gourmet » et le service de traiteur haut de gamme. Ces établissements proposent un menu de cuisine mexicaine traditionnelle et une déclinaison de tacos à base de garnitures d'inspiration « indigène » et des terroirs du sud. Les tacos sont classés dans la catégorie d'entrées ou hors-d'œuvre, tapas ou bocadillos, une sorte de petits en-cas, à base de maïs. Parmi les plats, en principe "populaires » et souvent méprisés, on trouve aussi la moelle à la braise ou des tacos aux vermicelles secs. Ces restaurants et quelques brasseries de haut de gamme, comme El Botanero (1999) et La Nacional (2003), proposent des tacos garnis au fromage parsemés d'insectes. Les insectes les plus utilisés pour la préparation de ces tacos sont les sauterelles, les chenilles et les larves de fourmis. Les restaurants " gourmets » s'en servent pour décorer les viandes ou les mixer avec du fromage. Dans une version plus sophistiquée, on trouve des tacos, appelés wraps, garnis au blanc de poulet, au saumon et à la viande grillée (bavette généralement). À part ces derniers, ces tacos sont en général uniquement servis en restaurant. Les tacos dits " gourmets » sont évidemment les plus coûteux. Les prix varient aux alentours de $\$ 50$ à \$100 pesos l'unité (alors que le prix moyen est de $6 \$$ pesos pour un taco acheté dans la rue). Les restaurants de style "gourmet» Zicatela (2001) et La Nacional (2003) proposent néanmoins une offre similaire, mais moins sophistiquée et plus économique. La "disposition ascétique» des consommateurs de tacos, désormais considérés "gourmets " car «authentiques » (Johnston \& Bauman, $2007: 198)$, participe à la légitimation des mets d'origine populaire. En les proposant, les restaurants les légitiment, de même que les connaisseurs qui les consomment, ce qui stimule leur diffusion aux autres classes sociales. La consommation de tacos ne semble pas forcément obéir à un "éclectisme culturel " (Peterson, 1992), mais plutôt à une valorisation de la cuisine du terroir et à une revendication de l'identité nationale ; ces deux derniers aspects se révèlent être, indépendamment de l'origine sociale des consommateurs, des sources de distinction sociale. La consommation éclectique des tacos n'est présente que chez les groupes sociaux favorisés. Celle-ci est le fruit d'une diversification gastronomique sélective et s'effectue de manière ponctuelle. 


\section{Les nouvelles scènes sociales des prises alimentaires}

23 s'effectuent les prises alimentaires. Il est donc important de tenir compte de la configuration sociale, à l'origine populaire, pour comprendre les effets de légitimation que les tacos ont connus dernièrement. C'est à partir du moment où les tacos sont entrés dans le monde de la restauration qu'ils sont devenus des « plats » plus légitimes. Leur légitimation est liée à l'adoption progressive de ce type de consommation par les groupes aisés dans un cadre plus formel.

24 L'univers des consommateurs de tacos est au départ issu des classes «populaires » et les clients faisaient généralement fi du contexte de consommation. Effectivement, la plupart des clients étaient des «consommateurs ambulants», occasionnels, des travailleurs ou des employés qui n'avaient pas les moyens de se rendre à leur domicile pour les repas traditionnels (Monnet \& Staszak, 2008). La distance entre le lieu de travail et le domicile a été l'une des principales variables ayant favorisé ce type d'alimentation. La disponibilité et le faible prix rendent les tacos séduisants aux yeux des consommateurs. Mais la configuration de consommation est aussi une contrainte. Celle-ci ne convient pas à tous les clients. Une partie de la clientèle a abandonné la consommation de rue, car porteuse de stigmates et connotée comme une pratique trop vulgaire. Cette clientèle achète des tacos à emporter ou se fait livrer chez soi. Un nouveau marché est apparu récemment : les tacos à domicile. Dans ce cas, le marchand installe ponctuellement une petite échoppe dans la propriété du commanditaire et sert directement les tacos sur place. L'hôte peut offrir à ses invités une dégustation de tacos " de rue » sans les connotations négatives associées à ce type de restauration, tout en évitant les inconvénients liés à la préparation d'un dîner. Cette formule, associée aux services de traiteur ${ }^{5}$, est en général très demandée pour les anniversaires, les réunions informelles, les lendemains de mariage, voire pour regarder les matchs de football à la télévision entre amis.

Cependant, les marchands de tacos ont développé une série de stratégies pour éviter la désaffection des clients. La principale a été de se sédentariser. C'est ainsi que les marchands se sont progressivement installés dans des locaux commerciaux créant les premières taquerias formelles, et ultérieurement les restaurants de tacos; et par la même occasion, les «tacos gourmets » ou gastronomiques. À partir de ce moment, la consommation a pu s'étendre à d'autres niches du marché. Plus conformes aux standards de la restauration classique, ces taquerias ont réussi à attirer un public plus diversifié. Au fur et à mesure que cette pratique s'est consolidée, elle a perdu ses stigmates « de classe ». Elles réduisent ainsi la crainte d'une consommation dont elle faisait l'objet. Les restaurants de tacos ne sont plus, ou sont, dans tous les cas, moins risqués et plus conformes aux normes de restauration.

Les pratiques alimentaires et les garnitures proscrites des «bonnes tables" dans le passé sont devenues des plats gourmets d'aujourd'hui. Citons le cas des insectes, la moelle braisée ou la barbacoa (viande cuite à l'étouffée qui est servie aussi bien dans la rue que dans les restaurants les plus exclusifs) ${ }^{6}$. D'autres ingrédients communs dans certaines régions côtières ont fait leur apparition dans un contexte inédit en tant que garnitures inhabituelles pour les tacos. C'est par exemple le cas des poissons et des fruits de mer. Les ragoûts du type «fait maison» figurent parmi les plus prisés des 
consommateurs (par exemple ceux proposés par Tacos Mode, Tacos El Fer et EcoTacos), alors que leur consommation par le passé était exclusivement réservée à l'espace domestique. Les garnitures à base de viande destinées à la préparation des tacos ont connu une montée en gamme significative, celles-ci vont de la traditionnelle bavette aux différents morceaux de choix. Un des exemples les plus éclairants est celui du restaurant Muelle 17 qui propose des tacos de langouste à $\$ 65$ pesos l'unité (4 euros); alors que les tacos de base ne coûtent que deux pesos l'unité (une quinzaine de centimes d'euro) pour les moins chers (haricots, pommes de terre ou viandes déchiquetées).

\section{Conclusion}

La consommation d'aliments sous forme de tacos est une pratique culturelle désormais largement partagée par tous les groupes sociaux à Monterrey. Elle est devenue emblématique de la culture culinaire locale. L'observation des pratiques alimentaires permet de mesurer le poids de l'appartenance sociale et son influence sur le choix des garnitures et les modalités de consommation. Bien que les tacos se soient diffusés dans tous les groupes sociaux, il existe en effet plusieurs modes de consommation. La localisation des lieux de consommation, les horaires et le contenu varient selon l'origine sociale des consommateurs. Cette différenciation sociale, observée au niveau local, semble être la condition qui a permis la généralisation de la consommation des tacos " populaires », au point de devenir plus largement un trait de la culture de la ville de Monterrey. Néanmoins, pour parler d'une revalorisation nationale de l'image du taco et des pratiques alimentaires populaires, d'autres recherches à plus grande échelle sont nécessaires.

Dans tous les cas, restituer les pratiques alimentaires dans leur contexte nous permet de voir que les tacos sont consommés par toutes les catégories socioprofessionnelles. En revanche, il ne s'agit pas d'un comportement culturel «omnivore» (Peterson \& Kern, 1996). Ce sont plutôt des pratiques alimentaires ponctuelles d'inspiration régionale qui participent à la définition de la cuisine nationale, et sont ajustées aux différentes positions sociales. La restructuration des rapports symboliques entre les classes entraîne une redéfinition de la légitimité culturelle en termes alimentaires. Cette redéfinition est aussi affectée par l'internationalisation de l'économie des biens culturels et l'adoption de nouveaux styles de vie (Coulangeon, 2011). Ces «métamorphoses » culinaires se sont traduites par l'enrichissement des garnitures, devenues plus sophistiquées, plus recherchées, surtout plus «nobles», et la modification des cadres de consommation, maintenant plus élégants et exclusifs, plus conformes aux standards internationaux de la restauration. La toile historique de fond, le relâchement des habitudes alimentaires et les nouveaux styles de vie expliquent la généralisation des pratiques alimentaires populaires à Monterrey. L'apogée récent des tacos peut être perçu comme une riposte culinaire et identitaire de la cuisine mexicaine face à la globalisation. Leur consommation est au demeurant un des lieux possibles où l'on peut observer la distinction sociale et une façon d'analyser la redéfinition de la légitimité culturelle. 


\section{BIBLIOGRAPHIE}

ABOITES Jaime, Breve historia de un invento olvidado. Las máquinas tortilladoras en México, México, UNAM, 1989.

ARELLANO Gustavo, Tacos USA : How Mexican Food Conquered America, USA, Scribner, 2012.

BAUER Arnold, « Millers and Grinders : Technology and Household Economy in Meso-America », Agricultural History, vol. 64, n 1, 1990, pp. 1-17.

BAUER Arnold, Goods, Power, History. Latin America's Material Culture, Cambridge, Cambridge University Press, 2001.

BOURDIEU Pierre, La distinction. Critique sociale du jugement, Paris, Minuit, 1979.

BRUEGEL Martin \& LAURIOUX Bruno (eds.), Histoire et identités alimentaires en Europe, Paris, Hachette, 2002.

COULANGEON Philippe, Les métamorphoses de la distinction. Inégalités culturelles dans la France d'aujourd'hui, Paris, Grasset, coll. « Mondes vécus », 2011.

GARCIA-GARZA Domingo, « Una etnografía económica de los tacos callejeros en México. El caso de Monterrey ", Estudios Sociales, vol. 19, n 37, 2011, pp. 32-63.

GARCIA-GARZA Domingo, «Prácticas alimenticias y clasificación social. ¿Los tacos son un alimento 'popular'? », Civitas, vol. 10, n. 3, 2010, pp. 430-449.

GARCIA-GARZA Domingo, L'entreprenariat informel. Le cas des marchands de tacos à Monterrey (Mexique), Paris, EHESS, thèse (dir. Remi Lenoir), 2009.

JOHNSTON Josée \& BAUMAN Shyon, « Democracy versus Distinction : A Study of Omnivorousness in Gourmet Food Writing ", American Journal of Sociology, vol. 13, n 1, 2007, pp. 165-204.

MONNET Jérôme \& STASZAK Jean-François, « Le consommateur ambulant : mobilités, stratégies et services ", Espaces \& Sociétés, vol. 4, n 135, 2008, pp. 19-24.

MONNET Jérôme, « L'ambulantage : Représentations du commerce ambulant ou informel et métropolisation », Cybergeo : European Journal of Geography, 17 octobre 2006a. Disponible en ligne : http : //cybergeo.revues.org/2683. DOI : 10.4000/cybergeo.2683.

MONNET Jérôme, « Le commerce de rue, ambulant ou informel et ses rapports avec la métropolisation : une ébauche de modélisation », Autrepart, vol. 3, n³9, 2006b, pp. 93-109.

NOVELO Victoria y GARCÍA Ariel, La tortilla. Alimento trabajo y tecnología, México, UNAM, 1987.

PETERSON Roger \& KERN R. M., « Changing Highbrow Taste : From Snob to Omnivore », American Sociological Review, vol. 61, n 5, 1996, pp. 900-907.

PETERSON Roger, « Understanding Audience Segmentation : From Elite and Mass to Omnivore and Univore ", Poetics, n³2, 1992, pp. 169-194.

PILCHER Jeffrey M., ; Que Vivan los Tamales! Food and the Making of Mexican Identity, Albuquerque, University of New Mexico, 1998.

PILCHER Jeffrey M., « ¡Tacos, Joven! Cosmopolitismo proletario y la cocina nacional Mexicana », Dimensión Antropológica, vol. 13, n 37, 2006, pp. 87-125.

PILCHER Jeffrey M., « Was the Taco Invented in Southern California? », Gastronomica : The Journal of Food and Culture, vol. 8, $n^{\circ} 1,2008$, pp. 26-38. 
PILCHER Jeffrey M., Planet Taco : A Global History of Mexican Food, New York, Oxford University

Press, 2012.

RIVRON Vassili, «Le goût de ces choses bien à nous : La valorisation de la samba comme emblème national (Brésil, années 1920-1940) », Actes de la recherche en sciences sociales, n 181-182, 2010, pp. 126-141.

SANCHEZ Sylvia, Pizza connexion. Une séduction transculturelle, Paris, CNRS, 2007.

SUREMAIN de, Charles, « Shawarmas contre McDo. Mondialisation et standardisation alimentaire à l'épreuve des contestations identitaires (Bolivie) ", Anthropology of food, 30 mai 2008. Disponible en ligne : http://aof.revues.org/index3693.html.

WARDE Alan \& MARTENS Lydia, Eating Out : Social Differentiation, Consumption and Pleasure, Cambridge, Cambridge University Press, 2000.

\section{NOTES}

1. Un taco est, selon la Real Academia de la Lengua Española, une «tortilla (galette) fourrée à la nourriture ". Les tacos sont donc à la fois un plat et une manière de consommer les aliments.

2. Par «patrimonialisation» on étend le processus de luttes pour l'obtention de la reconnaissance internationale par des institutions comme l'UNESCO qui a déclaré pour la première fois en 2010 (la cuisine traditionnelle mexicaine et les repas gastronomiques des Français) que la gastronomie d'un pays peut être considérée « patrimoine culturel immatériel de l'humanité » (1990).

3. Les taquerias sont au départ des échoppes ou stands mobiles de tacos. Ces derniers commencent à être vendus dans des établissements fixes dès lors que la Mairie de la ville de Monterrey restreint l'octroi de permis et que ces marchands cherchent à pérenniser leurs activités en échappant aux aléas liés à la vente ambulante.

4. La huitlacoche est un champignon noir pathogène qui pousse sur le maïs, considéré comme le caviar mexicain.

5. Le marché de restauration à domicile a explosé depuis une quinzaine d'années comme nous l'a avoué un enquêté. Plus récemment, à cause de l'insécurité liée aux vagues de violence imputée au trafic de drogues, le marché de livraison à domicilie à Monterrey est en pleine expansion.

6. Les groupes populaires ont tendance à apprécier certains morceaux cuits à la barbacoa (notamment la tête de veau et ses différentes parties : le palais, la langue, les yeux, la cervelle, les ris, etc.) ; alors que la joue de veau atteint un spectre social plus large allant jusqu'aux groupes les plus aisés. 


\section{RÉSUMÉS}

À partir de l'analyse du tacos, cet article étudie la diffusion des plats et des pratiques "populaires » à l'ensemble des milieux sociaux. La propagation de ce mode de consommation populaire ne fait pas pour autant disparaître ni les différences sociales ni les rapports symboliques entre classes, mais souligne plutôt la redéfinition de la légitimité des pratiques culturelles. Ainsi la revalorisation de la cuisine populaire obéirait à un ensemble de processus sociaux qui, tout en construisant une tradition culinaire nationale, participe également à maintenir les frontières symboliques entre les classes sociales.

A partir del análisis de los tacos, este artículo estudia la difusión de platos y prácticas " populares » a todos los grupos sociales. Esta forma de consumo no induce la desaparición por lo tanto de las diferencias sociales ni de las relaciones simbólicas entre las clases, sino subraya más bien la redefinición de la legitimidad de las prácticas culturales. Así, la revalorización de la cocina popular obedece a un conjunto de procesos sociales que, construyendo una tradición culinaria nacional, participa mantener las fronteras simbólicas entre las clases sociales.

Based on research concerning tacos, this article shows, how lower class meals and practices spread to all social milieu. Neither social differences nor symbolic relations among classes disappear through this type of consumption, which actually highlights the redefinition of legitimacy of cultural practices. Thus, this paper demonstrates how the consumption of tacos participates in the construction of a culinary national tradition, while at the same time maintaining symbolic frontiers between social classes.

\section{INDEX}

Mots-clés : Mexique, culture, distinction, alimentation, tacos, identité.

Keywords : Mexico, cultural practices, food, tacos, identity.

Palabras claves : México, prácticas culturales, alimentación, tacos, identidad.

\section{AUTEUR}

\section{DOMINGO GARCIA-GARZA}

Maître de conférences en civilisation latino-américaine et chercheur au CECILLE (Université Lille 3). Chercheur associé au CESSP équipe CSE (EHESS/Paris 1/CNRS).

Email : domingopointgarciagarzaatuniv-lille3pointfr 